\title{
A Narrative Review of Intensive Group Tobacco Treatment: Clinical, Research, and US Policy Recommendations
}

\author{
Chris Kotsen PsyD, CTTS ${ }^{1}$, Melissa L. Santorelli PhD, MPH², \\ Erika Litvin Bloom PhD ${ }^{3,4,5,}$, Adam O. Goldstein MD, $\mathrm{MPH}^{6}$, \\ Carol Ripley-Moffitt Mdiv, CTTS ${ }^{7}$, Michael B. Steinberg MD, MPH${ }^{8}$, \\ Michael V. Burke EdD ${ }^{9}$, Jonathan Foulds PhD ${ }^{10}$
}

'Tobacco Quitcenter, Lung Cancer Institute, Steeplechase Cancer Center, RWJ Barnabas Health, Robert Wood Johnson Barnabas Health University Hospital Somerset, Somerville, NJ; ${ }^{2}$ Community Health and Wellness Unit, New Jersey Department of Health, State of New Jersey, Trenton, NJ; ${ }^{3}$ Department of Psychiatry and Human Behavior, Warren Alpert Medical School of Brown University, Providence, RI; ${ }^{4}$ Department of Medicine, Warren Alpert Medical School of Brown University, Providence, RI; ${ }^{5}$ hhode Island Hospital, Warren Alpert Medical School of Brown University, Providence, Rl; ${ }^{6}$ Tobacco Intervention Programs, Department of Family Medicine, University of North Carolina, Chapel Hill, NC; ${ }^{7}$ Nicotine Dependence Program, Department of Family Medicine, University of North Carolina, Chapel Hill, NC; ${ }^{8}$ Rutgers Tobacco Dependence Program, Division of General Internal Medicine, Department of Medicine, Rutgers Robert Wood Johnson Medical School, New Brunswick, NJ; ${ }^{9}$ Nicotine Dependence Center, Mayo Clinic, Rochester, MN; ${ }^{10}$ Population Health and Cancer Control Program, Cancer Institute, College of Medicine, Penn State University, Hershey, PA

Corresponding Author: Chris Kotsen, PsyD, CTTS, Tobacco Quitcenter, Lung Cancer Institute, Steeplechase Cancer Center, RWJ Barnabas Health, Robert Wood Johnson University Hospital Somerset, Somerville, NJ, USA. Telephone: 908-685-

2902; E-mail: chris.kotsen@RWJBH.org

\begin{abstract}
Introduction: Clinical practice guidelines recommend comprehensive treatment for tobacco dependence including pharmacotherapies and behavioral interventions. Group counseling may deliver unique treatment aspects not available with other modalities. This manuscript provides a narrative review of group treatment outcomes from real-world practice settings and complements recent meta-analyses of randomized controlled trials (RCTs). Our primary goals were to determine whether group treatments delivered in these settings have yielded similar quit rates compared to individual treatment and to provide recommendations for best practices and policy.

Methods: Group treatment was defined as occurring in a clinical or workplace setting (ie, not provided as part of a research study), led by a professionally trained clinician, and offered weekly over several weeks. English language PubMed articles from January 2000 to July 2017 were searched to identify studies that included outcomes from both group and individual treatment offered in real-world settings. Additional data sources meeting our criteria were also included. Reports not using pharmacotherapy and research studies (eg, RCTs) were excluded. The primary outcome was short-term, carbon monoxide (CO)-validated point prevalence abstinence (4-week postquit date). Results: The review included data from 11 observational studies. In all cases, group treatment(s) had higher 4-week CO-validated quit rates (range: 35.5\%-67.3\%) than individual treatment(s) (range: $18.6 \%-53.3 \%$ ).
\end{abstract}


Conclusions: Best practice group treatments for tobacco dependence are generalizable from research to clinical settings and likely to be at least as effective as intensive individual treatment. The added advantages of efficiency and cost-effectiveness can be significant. Group treatment is feasible in various settings with good results.

Implications: A major barrier to achieving high rates of tobacco abstinence is under-utilization of evidence-based treatment interventions. This review demonstrates the effectiveness and utility of group treatment for tobacco dependence. Based on the available data described in this narrative review in conjunction with existing RCT data, group treatment for tobacco dependence should be established and available in all behavioral health and medical settings. Group tobacco treatment is now one of the mandated reimbursable tobacco treatment formats within the US health care system, creating enormous opportunities for widespread clinical reach. Finally, comprehensive worksite group programs can further extend impact.

\section{Introduction}

Tobacco use remains the leading cause of preventable death and disease in the United States. ${ }^{1}$ As of 2010 , an estimated $8.7 \%$ of US annual health care spending, or as much as $\$ 170$ billion per year, was attributable to smoking. ${ }^{2}$ Most people who use tobacco want to quit and engage in multiple quit attempts. ${ }^{3-6}$ Unfortunately, most quit attempts end in relapse. ${ }^{7}$ Clinicians routinely attempt to assist patients with overcoming cessation fatigue or tiredness in trying to quit tobacco. ${ }^{8}$ Low success in quitting and high relapse rates may partly be because only $31 \%$ of smokers making quit attempts use counseling and/or medication when trying to quit, which remains unchanged from 2005 to $2015 .{ }^{3}$ In fact, only $2.8 \%$ of patients making a quit attempt reported using one-on-one counseling and only $2.4 \%$ used a stop smoking clinic, class, or support group. ${ }^{3}$ Despite clear evidence-based research recommendations regarding the effectiveness of these tobacco treatments, there is a continuing need to evaluate and refine these recommendations as research is translated into practice. The latest US Public Health Service (PHS) Clinical Practice Guideline for Treating Tobacco Use and Dependence emphasizes optimal combinations of specialized behavioral and pharmacologic approaches. ${ }^{7}$ Medications primarily target cravings and components of addiction (eg, withdrawal symptoms), whereas behavioral approaches may modify psychosocial aspects of tobacco dependence, for example, helping patients establish new habits for managing tempting situations. Recommended formats for behavioral approaches include individual and group face-to-face counseling and telephone (eg, quitline) counseling. ${ }^{7}$

Theoretically, group-oriented tobacco treatment could foster significant behavior change through therapeutic factors such as instilling hope, universality, altruism, group cohesion, imitative behavior, and models of successfully coping with life without tobacco. ${ }^{9}$ Hajek et al. ${ }^{10}$ and Hajek ${ }^{11}$ presented data that group tobacco treatment aiming to maximize group processes such as patient interaction and intergroup discussion among participants impacted outcomes more strongly than a traditional didactic or "class" style of group tobacco treatment. Group-oriented treatment led to significantly higher short- and long-term quit rates, as well as better attendance. ${ }^{10}$

A long history exists of efforts to promote group tobacco treatment in the United States. Some patients reported using Smokenders group meetings in the 1970s, 1980s, and 1990s. Kanzler et al. ${ }^{12}$ reported that a 9-week Smokenders group program $(n=553)$ resulted in a self-reported, intent-to-treat 7-day point prevalence (PP) quit rate of $70 \%$ at the end of the program. Bakkevig et al. ${ }^{13}$ provided data from a randomized comparative trial of patients who received treatment from their general practitioner or attended an 8-week Smokenders group. At 2 months postquit date, $54 \%$ of Smokenders patients were smoke-free compared to $13 \%$ who saw their physician, based on self-report. During this same time period, the American Cancer Society and American Lung Association began promoting "Fresh Start" and "Freedom From Smoking" group classes, respectively. An earlier version of these programs, ${ }^{14}$ which did not include medications, reported 3-month PP quit rates at 24\%-29\%; however, these programs have not been recently evaluated.

Three relatively recent reviews of group treatment outcomes from randomized controlled trials (RCTs) have been published. First, the US Preventive Services Task Force recently gave group treatment a grade A recommendation, the strongest recommendation, primarily based on an earlier (2000) meta-analysis of 52 treatment arms reported in the PHS Guideline. ${ }^{715}$ In comparison to no treatment, group treatments produced an odds ratio (OR) of 1.3 $(95 \%$ confidence interval $[\mathrm{CI}]=1.1 \%$ to $1.6 \%)$, with an estimated long-term quit rate of $13.9 \%$. Individual treatments (67 arms) produced an OR of $1.7(95 \% \mathrm{CI}=1.4 \%$ to $2.0 \%)$ with an estimated $16.8 \%$ long-term quit rate. Second, Mottillo et al. ${ }^{16}$ conducted a meta-analysis of individual, group, and telephone counseling outcomes from 50 RCTs ( $N=26$ 927) conducted in Europe, North America, and Australia. All three formats were found to significantly increase smoking abstinence compared to controls, with ORs of $1.49(95 \% \mathrm{CI}=1.08 \%$ to $2.07 \%)$ for individual counseling, 1.76 (95\% CI $=1.11 \%$ to $2.93 \%)$ for group counseling, and $1.58(95 \%$ $\mathrm{CI}=1.15 \%$ to $2.29 \%$ ) for telephone counseling. Finally, a 2017 Cochrane review concluded that group treatment has better outcomes than no intervention, self-help programs, or receiving brief support from a health care provider. ${ }^{17}$ Also, this review reported that no differences in quit rates were found in six RCTs comparing group counseling and individual counseling approaches. ${ }^{17}$

In the United States, the Affordable Care Act now mandates group tobacco treatment as a covered preventive benefit by Medicaid and most private health insurance plans. ${ }^{18}$ Given that there has been no increase in the uptake of professionally assisted tobacco treatment utilization in the last decade, ${ }^{3}$ and given that there have been no recent comprehensive narrative reviews of group tobacco treatment outcomes from real-world clinical practice settings (ie, the treatment that patients received was not part of a clinical trial), this manuscript complements the reviews of RCTs described earlier and provides a new narrative review of recent group treatment outcomes in realworld clinical settings for practitioners, public health professionals, and researchers. In this narrative review, we provide a qualitative 
and quantitative assessment of findings across heterogeneous observational studies, including different clinical settings, clinician types, populations, and outcome measures. We conducted a narrative review $^{19,20}$ because this approach can help clinicians and researchers in identifying and integrating clinical and setting treatment themes, to close practice gaps observed across studies, and to generate hypotheses for future research. We have restricted our policy recommendations to the United States, given our familiarity with the US health care system (six authors are practicing clinicians in the United States) and the significant differences between the US health care system versus other countries.

We had three primary aims for our review. Our first aim was to compare group treatment quit rates to similarly intensive individual treatment quit rates in real-world clinical settings in which both types of treatment were available. A second aim was to compare group and individual treatment results in real-world clinical practice settings to outcomes from traditional research studies (ie, RCTs), given often significant differences between treatment delivered in the context of research studies (ie, exclusion of patients with medical and behavior health comorbidities, ${ }^{21}$ treatment delivered by specially trained research staff, participants complete extensive research assessments and are paid for participation/travel, participants randomly assigned to treatment condition) and treatment delivered in real-world clinical practice settings (ie, inclusion of all tobacco users who want to quit, patient may choose group vs. individual). Finally, we sought to elucidate contemporary best clinical practices for comprehensive group tobacco treatment.

\section{Methods}

Group treatment was defined as occurring in a real-world clinical practice or workplace setting (ie, not in the context of a research study), led by a professionally trained clinician, and offered weekly for at least several weeks to groups of more than two participants. Given that there is strong evidence that combining counseling with medication results in better outcomes than either counseling or medication alone, ${ }^{7}$ and that many real-world practice settings routinely use multiple, over-the-counter (OTC) quitting medications, ${ }^{22,23}$ we restricted our review to treatment provided after the year 2000, when the nicotine lozenge, a popular medication, was more widely available OTC (eg, nicotine lozenge was approved to be sold OTC in mid-1999 in the United Kingdom and in late 2002 in United States).

\section{Search Strategy}

English language PubMed articles from January 2000 to July 2017 were searched using the following search string: ((smoking[Title] OR tobacco[Title]) AND group[Title/Abstract]) AND (session[Title/ Abstract] OR class[Title/Abstract] OR therapy[Title/Abstract] OR psychotherapy[Title/Abstract] OR counseling[Title/Abstract] OR treatment[Title/Abstract]) AND (“2000/01/01”[PDAT]: “3000/12/31"[PDAT]) AND "humans"[MeSH Terms] AND English[lang] AND "adult"[MeSH Terms] NOT Randomized Controlled Trial[ptyp]. Two authors independently reviewed the abstracts of all articles identified by this search. Articles were selected based on predetermined inclusion and exclusion criteria (Table 1). A third author was available to resolve any discrepancies. Reference lists of included articles were also searched for additional data sources that met our criteria but were not identified by the PubMed search. Finally, other real-world data sources that were already known to the authors were included if they met our criteria and were deemed to potentially contribute to the understanding of group treatment best practices. If data were presented only in ORs, authors were contacted to provide sample sizes and short-term quit rates whenever possible.

\section{Review Outcomes}

Our primary outcome of interest was short-term, carbon monoxide $(\mathrm{CO})$-validated PP quit rate at 4 weeks after the target quit date. Also, when possible, similar to the work of Roberts et al., ${ }^{24}$ we reported on PP quit rates at the last follow-up if it was after 4 weeks.

\section{Results}

The PubMed search initially returned 718 articles. Many articles at this stage were eliminated because they were not relevant (ie, focus group studies, hypnosis, acupuncture, e-cigarettes, financial incentives, texting, quitline, etc.). After abstract review, 31 full articles were selected for further review and 6 articles were included in our review. Many of the articles excluded at this second stage described brief single visit treatment; did not provide data comparing multisession, one-on-one formats versus group treatment; did not include quitting medications; and/or did not report amounts of medications used or prescribed. Three additional data sources from real-world practice settings were identified by reviewing the references from identified articles. Finally, data from two additional real-world data

Table 1. Study Inclusion and Exclusion Criteria

Inclusion criteria

1. Observational studies or treatment delivered in a real world clinical practice setting (ie, not in the context of a research study) and included patients in both group treatment and individual treatment

2. Patients were motivated to quit and practice settings accepted all patients seeking treatment

3. Counseling was moderate to high intensity (ie, at least weekly visits)

4. US FDA-approved medications were used by at least $70 \%$ of patients

5. Outcomes were reported at 4-week postquit date (CO validated, if possible)

6. Quit rates were reported using 7- or 14-day point prevalence abstinence

7. Intent-to-treat analyses were conducted (patients lost to follow-up were included in the denominator when calculating quit rates)
Exclusion criteria

1. Randomized controlled trial (RCT) or treatment delivered in the context of a prospective research study

2. Some patients were excluded because of comorbidities (eg, medical, psychiatric, and/or active alcohol or illicit drug use)

3. Treatment was brief or provided in a single visit

4. US FDA-approved medications were not used or reported

$\mathrm{CO}=$ carbon monoxide; FDA = Food and Drug Administration. 
Table 2. Studies Included in the Review

\begin{tabular}{|c|c|c|c|c|c|c|c|}
\hline \multirow[b]{2}{*}{ Author (year) } & \multicolumn{2}{|c|}{ Group } & \multicolumn{2}{|c|}{ Individual } & \multirow[b]{2}{*}{$\begin{array}{c}\text { Setting } \\
\text { (\% non-White) }\end{array}$} & \multirow[b]{2}{*}{ Clinician type } & \multirow[b]{2}{*}{ Medications } \\
\hline & $\begin{array}{l}\text { Sample } \\
\text { size }\end{array}$ & $\begin{array}{l}\text { Quit } \\
\text { rate* }(\%)\end{array}$ & Sample size & $\begin{array}{c}\text { Quit } \\
\operatorname{rate}^{a}(\%)\end{array}$ & & & \\
\hline Judge (2005) & 321 & 57 & 6523 & 53.3 & England (3.2) & Smoking cessation advisor & NRT; bup \\
\hline McEwin (2006) & 822 & 42 & 679 & 32 & England (12) & $\begin{array}{l}\text { Clinic cessation specialists }(\mathrm{G}) \text {; } \\
\text { community pharmacists or PC } \\
\text { nurses (I) }\end{array}$ & NRT; bup \\
\hline Bauld (2006) & 448 & 44.4 & $11126^{\mathrm{b}}$ & 20.2 & Scotland $(0)$ & $\begin{array}{l}\text { Smoking cessation advisor }(\mathrm{G}) \text {; } \\
\text { community pharmacists }(\mathrm{I})\end{array}$ & $\begin{array}{l}\text { NRT; bup }(G) ; \\
\quad \text { NRT }(\mathrm{I})\end{array}$ \\
\hline Bauld (2009) & 411 & 35.5 & 1374 & 18.6 & Scotland (NR) & $\begin{array}{l}\text { Community cessation } \\
\text { specialists }(\mathrm{G}) \text {; network } \\
\text { pharmacist or pharmacy } \\
\text { assistant }(\mathrm{I})\end{array}$ & $\begin{array}{l}\text { NRT; bup; var }(G) \text {; } \\
\text { NRT (I) }\end{array}$ \\
\hline Brose (2011) & $8966^{\mathrm{cdd}}$ & 49.9 & $114672^{\mathrm{c}, \mathrm{e}}$ & 35.4 & England (14.8) & $\begin{array}{l}\text { Trained advisors in PC; } \\
\text { specialist clinics; pharmacy; } \\
\text { other settings }\end{array}$ & NRT; var; bup \\
\hline Mardle (2012) & $318^{\mathrm{f}}$ & 67.3 & 870 & 50.7 & England (NR) & $\begin{array}{l}\text { PCT-led specialist teams; } \\
\text { community-based health } \\
\text { care providers }\end{array}$ & NRT; var; bup \\
\hline Hiscock (2013) & $\begin{array}{l}3844(\mathrm{o}) \\
2145(\mathrm{c})\end{array}$ & $\begin{array}{l}50.8(\mathrm{o}) \\
49.1(\mathrm{c})\end{array}$ & $123321^{\mathrm{e}}$ & 33.9 & England (11.8) & $\begin{array}{l}\text { Specialist; GP; nurse; health care } \\
\text { assistant; pharmacy staff }\end{array}$ & NRT; bup; var \\
\hline Dobbie (2015) & $\begin{array}{l}4780^{\mathrm{g}}(\mathrm{o}) \\
2512^{\mathrm{g}}(\mathrm{c})\end{array}$ & $\begin{array}{l}52.1 \\
50.1\end{array}$ & $\begin{array}{c}140119^{\mathrm{g}} \\
24736^{\mathrm{g}} \text { (drop-in) }\end{array}$ & $\begin{array}{l}33.8 \\
33.3\end{array}$ & England (10.7) & $\begin{array}{l}\text { Specialist; GP; nurse; } \\
\text { health care assistant; } \\
\text { pharmacy staff }\end{array}$ & NRT; bup; var \\
\hline $\begin{array}{l}\text { McAlpine } \\
(2015)\end{array}$ & 145 & 61.4 & $338^{e}$ & 50.9 & England (26) & Specialist & NRT; bup; var \\
\hline Foulds (2006) & $448^{\mathrm{h}}$ & 57.6 & $229^{i}$ & 47.7 & $\begin{array}{c}\text { United States } \\
\qquad(34.4)\end{array}$ & Specialist & NRT; bup \\
\hline $\begin{array}{r}\text { Santorellii } \\
(2015)\end{array}$ & $212^{\mathrm{i}, \mathrm{k}}$ & 59.0 & $1427^{\mathrm{j}, \mathrm{k}}$ & 46.0 & $\begin{array}{c}\text { United States } \\
\qquad(33.8)\end{array}$ & Specialist & NRT; bup \\
\hline
\end{tabular}

bup = bupropion; $\mathrm{c}=$ closed $; \mathrm{G}=$ group; $\mathrm{GP}=$ general practitioner $; \mathrm{I}=$ individual, $\mathrm{NR}=$ not reported NRT = nicotine replacement therapy; $\mathrm{o}=$ open; $\mathrm{PC}=$ primary care; $\mathrm{PCT}=$ Primary Care Trust var $=$ varenicline.

${ }^{a}$ Quit rates are 14-day point prevalence abstinence for England and Scotland studies and 7-day point prevalence abstinence for US studies.

'One-to-one individual treatment with pharmacist.

'Raw data provided by L. Brose (personal communication, January 4, 2017).

dIncludes both open and closed group.

'Includes individual drop-in.

Includes open, closed, and workplace groups.

gIncludes some overlapping patient data from Hiscock et al..$^{26}$

hIncludes those who attended at least one group session.

Includes those who attended at least one individual session.

In addition to the sample shown for group only and individual only, another 888 patients received both group and individual (quit rate of $62.1 \%$ ) treatments.

${ }^{k}$ Includes some overlapping patient data from Foulds et al. ${ }^{27}$

sources already known to several of the authors were included for 11 observational studies meeting criteria for this review (Table 2). Sample sizes listed in Table 2 may not match the total Ns reported in the text because some samples included patients who did not receive group or individual treatment. Although we did not restrict our literature search to any specific location or country, all studies that met our criteria were from one of two countries: the United Kingdom $(N=9)$ or the United States $(N=2)$. Therefore, we decided to group them by country (United Kingdom vs. United States) given the significant differences in the health care systems between those two countries. Outcomes from both countries were based on quit rates at 4 weeks after the target quit date. However, UK outcomes followed the Russell Standard ${ }^{25}$ of 14-day PP ("Have you used any tobacco in the last 14 days, even a single puff?"), whereas US studies reported 7-day PP ("Have you used any tobacco in the last 7 days?").

\section{UK Studies}

All UK sources reported on individuals who used the Stop Smoking Services (SSSs), offered through the single payer National Health Service, with locations throughout the United Kingdom. The National Health Service SSS, established in 1999, provides both behavioral support (individual and/or group) by trained smoking cessation advisors and medication. ${ }^{28}$ Advisors can be specialists whose job consists only of providing smoking cessation services, or other health care providers (eg, pharmacists, physicians) who provide smoking cessation services as part of their job. Specific programs and medications available have changed over time and vary by location.

Judge et al. ${ }^{29}$ reported on outcomes of nearly 7000 patients who used SSS between 2001 and 2003 in: (1) Nottingham, an ethnically diverse city in the East Midland region with many areas of low socioeconomic status (SES) and (2) North Cumbria, a rural area in the northwest. Patients typically received 5 weeks of counseling, starting 
1 week before quitting and continuing 4 weeks after a target quit date. Most patients (about 70\%) received nicotine replacement therapy (NRT), with $18 \%-20 \%$ receiving bupropion and $2 \%-3 \%$ combined NRT and bupropion. The CO-validated 4-week quit rate was $53.3 \%$ in individual versus $57.0 \%$ in group treatment. In a multivariate analysis, group treatment demonstrated a significantly higher CO-validated quit rate, $\mathrm{OR}=1.38(95 \% \mathrm{CI}=1.09 \%$ to $1.76 \%)$ compared to individual treatment. Primary limitations of this study are the small proportion of patients who received group treatment $(n=321)$ versus individual treatment $(n=6523)$, and no information reported on the equivalence of dosing (ie, minutes of counseling) between individual and group treatments. Rural and semirural areas had lower group treatment turnout.

McEwen et al..$^{30}$ compared group treatment provided by specialist counselors receiving ongoing supervision $(n=822)$ versus individual treatment offered by Community Advisors, mostly pharmacists and primary care nurses, $(n=679)$ in the outer boroughs of London between 2001 and 2003. Counseling content was "withdrawaloriented" and nearly identical in both treatment formats. Groups consisted of 30-60 patients and met weekly for 6 weeks, with each meeting up to 2 hours. Individual treatment included six weekly sessions of about 30 minutes per visit. Most patients (75\%) used NRT, with $22 \%$ using bupropion. CO-validated 4 -week quit rates were higher in group treatment $(42 \%)$ than individual treatment $(32 \%)$. The difference in the odds of quitting remained significant even after controlling for possible confounding factors, such as level of dependence and patient demographics such as race and ethnicity and SES $(p<.001)$.

Bauld et al. ${ }^{31}$ reported on group treatment $(n=448)$ and individual treatment $(n=11$ 126) outcomes in Scotland from 2004 to 2005 , with a high number of low-income tobacco users. Offered in multiple venues (eg, health center, other community venues), group treatment included seven weekly visits, with the quit date at the third week. Both medical and nonmedical professionals who had received specialist training delivered the group treatment. Almost all patients in group treatment used NRT; very few $(<20)$ used bupropion. Individually treated patients received services in 167 participating pharmacies. All patients in individual treatment received NRT (16hour patch). Pharmacists or pharmacy assistants provided counseling for at least five weekly sessions with the quit date at week 1. Patients remaining in the program at week 5 could continue for up to seven additional weeks. Often there was no privacy, with counseling occurring at the counter where clients received patches. Many patients received a longer first session with the pharmacist, but subsequent visits were often very short (eg, about 5 minutes). Overall, this pharmacy treatment format may have been more of a brief-tomoderate intensity intervention, compared to the longer duration of group treatment counseling. The CO-validated quit rate at 4 -week follow-up was $44.4 \%$ for group treatment versus $20.2 \%$ for pharmacy treatment. These two treatment models were set up differently and were not meant to be compared directly. For example, pharmacy patients initially set a quit date and received NRT at their first visit, but many did not return for a second visit. Thus, less motivated patients may have been included in the intent-to-treat quit rates, in comparison to patients who received group treatment. The pharmacy later introduced an additional introductory visit ("week zero"), with NRT provided at the second visit. Additionally, another study limitation was that patients needed to fail the patch before trying other medications.
Bauld et al..$^{32}$ conducted another analysis of group $(n=411)$ versus pharmacy-provided individual $(n=1374)$ treatment in Glasgow, Scotland, in 2007; both groups included many with low SES. Group treatment participants met in 1-hour weekly sessions for 7 weeks. Individual treatment patients received an initial assessment ( $>15$ minutes) followed by 12 weekly visits (5-15 minutes). Patients in the group treatment received NRT (84\%), bupropion $(3 \%)$, or varenicline $(13 \%)$, whereas all patients in the individual treatment received access to 16-hour nicotine patch (100\%). Again, the CO-validated quit rate at 4 weeks was higher for the group treatment $(35.5 \%)$ versus the individual treatment $(18.8 \%)$. This difference was significant after controlling for a range of participant characteristics (ie, level of addiction and SES) and pharmacotherapies used $(\mathrm{OR}=1.98,95 \% \mathrm{CI}=1.50 \%$ to $2.62 \%)$. As with the prior report, individual treatment may have been less intensive than group treatment.

Brose et al..$^{33}$ provided retrospective data from 126890 treatment episodes in 24 SSS locations in the United Kingdom between 2009 and 2010. Treatment formats included intensive individual and group treatment in primary care, specialist clinics, and pharmacy settings. About $88 \%$ of patients used medications (varenicline, bupropion, or NRT), with specialist clinics more likely to prescribe combination NRT or varenicline for highly dependent patients. Controlling for a number of client characteristics (ie, race and ethnicity, SES, addiction severity) and intervention variables, both closed and open (ie, rolling admission) group treatments had higher 4-week CO-validated quit rates compared to individual treatment $(\mathrm{OR}=1.43,95 \%$ $\mathrm{CI}=1.16 \%$ to $1.76 \%$ for closed $\mathrm{OR}=1.46,95 \% \mathrm{CI}=1.19 \%$ to $1.78 \%$ for open). Raw data provided (L. Brose, personal communication, January 4, 2017) showed that closed groups had a $49 \%$ quit rate and open groups had a quit rate of $50.6 \%$, compared to individual treatment (including drop-ins) quit rate of $35.4 \%$

Mardle et al. ${ }^{34}$ conducted an analysis of SSS provided through three Primary Care Trusts (PCTs) in England (Bournemouth and Poole, South East Essex, and Warwickshire) in 2008. Group treatment was provided by a PCT specialist team in one setting. Another PCT provided treatment by PCT-led specialist teams, including a mix of a PCT-led specialist team, primary care practitioners, pharmacists, dentists, and opticians. One of the PCTs offering groups had three options (closed, rolling, and workplace groups), whereas the other only offered closed groups. In all three PCTs, the majority of participants used NRT $(55.8 \%-65 \%)$, with $24.5 \%-34.3 \%$ using varenicline and $3 \%-4 \%$ using bupropion. Once again, group treatment resulted in higher CO-validated 4 -week quit rates $(54.9 \%-76.2 \%)$ versus individual treatment $(42.6 \%-51.2 \%)$, but the data did not control for client factors such as level of motivation. The authors suggested that service structure, treatment format, health care professional involved, and pharmacotherapy all played a role in the variable quit rates. Although closed groups had the highest quit rates, it was noted that patient choice and tailoring of patient needs are key treatment factors. It was also noted that group treatment was not feasible in many rural areas.

Hiscock et al., ${ }^{26}$ in a retrospective study, provided CO-validated 4-week quit rate data for 132586 quit attempts by patients aged 19-59 in England who accessed National Health Service SSS between 2010 and 2011. Most patients were treated by specialists, general practitioners, nurses, health care assistants, or pharmacists. More than half were seen by specialist clinicians. Nearly $79 \%$ reported using medications (single NRT, combination NRT, bupropion, varenicline, or a mixture). The quit rate for individual treatment was 
$33.9 \%$ versus $50.8 \%$ for open groups and $49.1 \%$ for closed groups. In a multivariate analysis, controlling for some patient characteristics (race and ethnicity and SES), open group treatment was more successful than individual counseling $(\mathrm{OR}=1.26,95 \% \mathrm{CI}=1.12 \%$ to $1.41 \%$ ) for most clients (not including prisoners and the longterm unemployed).

Dobbie et al. ${ }^{28}$ conducted an analysis of retrospective data from 49/150 SSS centers ( $N=202804$ cases) from July 2010 to June 2011in England. The dataset overlapped with data presented by Hiscock et al. ${ }^{26}$ Whereas Hiscock et al. ${ }^{26}$ restricted their analysis to patients aged 19-59, Dobbie et al. ${ }^{28}$ included patients of all ages. Almost all patients received medication, with a significant proportion receiving varenicline and the rest NRT (single or combination), and patients who saw specialist practitioners had higher quit rates than patients who saw other types of practitioners. In the analysis, most patients $(n=140119,79 \%)$ received individual treatment, whereas only $2.7 \%(n=4780)$ received open group treatment, and $1.4 \%(n=2512)$ received closed group treatment. However, the $\mathrm{CO}$-validated 4 -week quit rate was $33.8 \%$ for individual treatment, $52.1 \%$ for open groups, and $50.1 \%$ for closed groups. Open groups were significantly more effective than individual treatment ( $\mathrm{OR}=1.28,95 \% \mathrm{CI}=1.15 \%$ to $1.41 \%)$.

Finally, McAlpine et al. ${ }^{35}$ conducted an analysis of 516 patients seen at a single specialist clinic (Whitechapel) in London from 2013 to 2014, representing a higher proportion of males or ethnic minorities than the national average. Treatment typically included seven weekly sessions in individual or group (closed or open) format. Nearly all patients received pharmacotherapy, with $49 \%$ receiving NRT and $47 \%$ receiving varenicline. The 4 -week, CO-validated quit rate was higher for patients who received closed group tobacco treatment $(61.4 \%)$, compared to individual treatment $(50.9 \%)$.

\section{US Studies}

Only two US studies meeting criteria were identified in the United States. Due to the complex health care system and billing difficulties with face-to-face counseling, ${ }^{36,37}$ group treatment is not widely available in the United States. Many tobacco users in the United States may only receive brief physician advice and/or some quitline support.

Foulds et al. ${ }^{27}$ conducted a retrospective cohort study involving 1021 patients who received treatment at the Tobacco Dependence Program at the University of Medicine and Dentistry of New Jersey (UMDNJ) School of Public Health. This specialist clinic has provided free treatment since 2001. The percent of racial-ethnic minorities who received treatment was similar to the proportion of racial-ethnic minorities in the population of New Jersey in 2007.38 Treatment included an initial 50-75 minutes assessment followed by individual or group counseling. Group treatment consisted of six weekly 90-minute group sessions with the target quit date at week 2. Patients also received free and/or discounted OTC NRT and prescription medications (bupropion, nicotine inhaler, and nicotine nasal spray). Overall, $87 \%$ reported using medications. After controlling for other significant patient predictors (SES and addiction severity), patients who attended at least one group treatment meeting $(n=448)$ had higher self-reported quit rates (7-day PP) at 4 weeks, compared to those who attended only individual treatment visits $(n=279), 57.6 \%$ versus $47.7 \%, p=.04$. This difference remained significant at 6 -month postquit $(41.3 \%$ vs. $28.7 \%, p=.02)$. A limitation of the study is that only $39 \%$ of patients were seen in person at 4-week follow-up and therefore able to provide a CO sample. Therefore, some of the patients' self-report was not biochemically validated. However, $99.2 \%$ of the patients who self-reported abstinence and had their CO measured had CO less than 10 ppm, validating their self-report.

An evaluation of a state-wide program funded by the New Jersey Department of Health and Senior Services describes outcomes from patients $(N=4975)$ enrolled in individual and group face-to-face treatment at 15 hospital-based programs at New Jersey Quitcenters from 2001 to $2006 .{ }^{39}$ The percent of racial-ethnic minorities who received treatment was similar to the proportion of racial-ethnic minorities in the population of New Jersey in 2007. ${ }^{38}$ Patient costs were free or minimal, including some free and discounted OTC NRT. The average patient was moderately dependent on tobacco and attended 5.4 treatment visits, including the intake. The treatment visits were either individual or group counseling, or a combination of individual and group treatment. Breath CO testing occurred at each visit. Most patients (79\%) used at least one US Food and Drug Administration-approved medication. Group treatment visits were either in structured, closed 6-week group programs or rolling, open group meetings, depending on treatment setting. In addition, over $75 \%$ of participants rated program satisfaction very high. The vast majority were treated at the clinic-based programs, with a minority treated at worksite programs and/or community settings in a closed group treatment format. The overall intent-to-treat quit rate at 6 months postquit date was $24.4 \%$, but the report did not distinguish between the types of counseling received or short-term quit rates.

A subsequent analysis of the same New Jersey Quitcenter data conducted by the New Jersey Department of Health (M. Santorelli, personal communication, 2015) determined that among those completing an intake, nearly $46 \%$ participated in at least one individual counseling session following the intake, and nearly $22 \%$ participated in at least one group counseling session. The average duration of individual counseling sessions was 49 minutes and, among patients who participated, the average number of individual visits completed by the 4-week follow-up was 2.1 (excluding intake meeting). The average duration for group counseling sessions was 80 minutes, and participants averaged 4.1 visits, indicating that typically these patients received more overall treatment exposure time. Furthermore, the 4-week intent-to-treat quit rate (7-day $\mathrm{PP}$ ) was $59 \%$ for patients who only received group treatment versus $46 \%$ for those who only received individual counseling. Those who attended individual and group meetings combined reported a short-term quit rate of $62.1 \%$. Six-month intent-to-treat quit rates were $45.7 \%$ for patients participating in group treatment, $37.8 \%$ for patients in individual counseling, and $49.6 \%$ for patients who received both individual and group treatments.

It should be noted that there was some overlap in Foulds et al. ${ }^{27}$ data published in 2006 and this subsequent analysis of New Jersey data, because the clinic associated with the Foulds et al. ${ }^{27}$ study was one of the 15 networks of Quitcenters, which all used similar databases to measure patient demographics and quit outcomes. These programmatic outcomes are compelling, but they are limited in that patient abstinence was not independently evaluated in all cases, because CO testing validated self-report only when available. Although there were limitations in the data, more than $99 \%$ of patients (630/634) who reported quitting had CO testing less than $10 \mathrm{ppm}$, validating their self-report.

\section{Discussion}

Recent reviews of RCTs have revealed that group-based treatment for tobacco dependence appears to be at least as efficacious 
as individual counseling treatment. Given that group treatment is now a mandated covered health insurance benefit under the US Affordable Care Act, and that real-world clinical practice settings may differ from research settings, we conducted a narrative review of group treatment outcomes in real-world practice settings, which were available from the United Kingdom and the United States In these settings, group treatments resulted in higher quit rates than individual treatments, suggesting that best practice group treatments are generalizable and can be transferred from research to clinical settings. Our results support the hypothesis that group treatment is at least equal to or possibly more effective than individual treatment.

Overall, the group quit rates observed in these real-world practice settings are consistent with and may be higher than the estimated quit rates from RCTs reported in prior meta-analyses. High quit rates in these settings show the benefits of strong integration and dose of treatment (medications plus intensive counseling), often in a "one stop shop" where patients received medications and counseling in the same physical location. Our review also indicates that both open and closed groups can produce similarly high quit rates. In particular, open or rolling admission groups may capitalize more on patient motivation, because these patients can immediately enter treatment.

A clear pattern in studies emerged that group treatment is more feasible in more populated areas. Many rural or semirural settings had a stronger preference for one-on-one treatment and significantly lower attendance at group treatment programs. Also, more treatment contact visits were associated with higher short-term quit rates, likely at least partly due to increased medication compliance. For example, Foulds et al. ${ }^{27}$ reported that patients attending at least six more visits had a $69.9 \%$ quit rate. Similarly, in a prospective study by Dobbie et al., ${ }^{28}$ six patient visits lead to a quit rate of $65.3 \%$. Novel engagement strategies for maximizing attendance appear to involve combinations of key programmatic factors: free medication(s) scheduling more treatment visits ${ }^{40}$ around the quit date, and "re-recruiting" smokers with slips or relapses. These types of interventions also led to high short- and long-term quit rates in the Lung Health Study. ${ }^{41-43}$ Additionally, moderate- and longer-term quit rates were achieved with similar comprehensive intensive group interventions by Hall et al. ${ }^{44,45}$

\section{Limitations}

Many potential complexities may exist in the interpretation of these clinical reports. First, clinicians leading groups (often tobacco treatment specialists) may have had more experience than clinicians providing individual counseling (eg, pharmacists), and these more experienced clinicians may be the causal agents accounting for higher quit rates in group treatment. More recently, Song et al. ${ }^{46}$ reported higher longer-term quit rates from specialist services compared to nonspecialist services, suggesting that more extensive training, supervision, and adherence to evidence-based practices lead to better outcomes. It is also possible that more experienced specialist clinicians are able to help patients correctly use medications or manage medication side effects so that medication adherence improves, as indicated by Dobbie et al. ${ }^{28}$

Another limitation, which applies to all outcomes from practice settings, is that patients were not randomly assigned to group versus individual treatment as in RCTs. In some reports, it was not clear how patients were allocated to group versus individual treatment and may have depended on availability, whereas in some cases patients self-select group or individual treatment modality. Individuals who choose group treatment may have different characteristics than those who choose individual treatment (eg, demographics, personality, and motivation). These characteristics may be independently associated with likelihood of quitting, regardless of the type of counseling received, and only some of the studies discussed here tried to control for baseline characteristics. It may be possible that in some studies, patients with more severe addictions, lower SES, and/or more severe psychiatric and/or substance use disorder comorbidities were steered by clinicians into individual treatment for clinical reasons more frequently, which could potentially bias the results in favor of group outcomes. On the other hand, five of the studies revi ewed $^{30,32,33,26,27}$ that did control for many of these patient characteristics, particularly SES and severity of addiction, still found group treatment quit rates to exceed one-on-one formats. Additionally, the Brose et al. ${ }^{32}$ discussed that the most successful intervention (group treatment) tended to have more severely dependent tobacco users, suggesting that the clients with more complexities are not necessarily choosing individual treatment. Finally, although our review included hundreds of thousands of patients, there is a risk of potential bias in our review, because we limited our database search strategy to PubMed and studies published in English.

\section{Research Recommendations}

The results of our review suggest several directions for future research. First, research should focus on identifying active group treatment mechanisms, possibly through analysis of behavior change techniques $^{47-49}$ and optimal group characteristics. An example includes exploring the impact of clinician interventions of prompting group interaction between group members. Another key example may be coding how patients were instructed in the correct use of quitting medication and/or how the clinician prompted intergroup dialogue on correct medication use (eg, intergroup discussion between veteran and novice group members). This may be critical, because combination medication is now more common in practice settings, ${ }^{23}$ with seven US Food and Drug Administration-approved medications often used in different combinations (and different dosages) with patients with more comorbidities and higher levels of dependence. Another example includes examining the impact of ongoing intensive clinical supervision provided to tobacco treatment specialists on ensuring higher quit rates for group treatment and accurately examining the impact of treatment fidelity as suggested by Song et al. ${ }^{46}$

Siu et al., ${ }^{15}$ referring to earlier PHS Guidelines, ${ }^{7}$ suggested that cessation rates might plateau after 90 minutes of total counseling contact time. On the other hand, in reviewing newer data, Siu et al. ${ }^{15}$ also noted that the largest effect was found in interventions that provided eight or more visits (although this was not significant). It was also noted that more or longer visits (ie, $\geq 20$ minutes/visit for individual treatment) led to higher quit rates. This may appear to be confusing to clinicians, because optimal time amounts for group and individual counseling may differ, but appear to have been combined in earlier meta-analytic studies. Future research should determine the optimal clinical "dosage of counseling" (ie, the number and duration of visits) and the ideal group size range. For example, commenting on a trend for studies with longer or more visits to lead to higher quit rates, Stead and Lancaster ${ }^{50}$ noted a clear difference in ORs in earlier PHS Guidelines between 2 and 3 visits, compared with more than 8 visits $(\mathrm{OR}=1.4,95 \% \mathrm{CI}=1.1 \%$ to $1.7 \%$ vs. $\mathrm{OR}=2.3,95 \%$ $\mathrm{CI}=2.1 \%$ to $3.0 \%)$. Again, these studies were earlier meta-analytic studies (2000) and did not break out format by individual or group, so optimal group dosing remains unclear in contemporary real-world 
practice settings. Future studies (ie, observational reports, RCTs, or prospective effectiveness studies) could assist in clearly indicating counseling time amounts delivered for individual and group counseling, to assist in comparisons of counseling dosing across studies.

The newer data in our review seem consistent with recent recommendations of Public Health England, ${ }^{51}$ indicating that of all treatment formats, group treatment (6-12 visits) plus pharmacotherapy had the highest effectiveness and largest effect size. Our review synthesis provides additional data supporting more intensive and comprehensive treatments ( $>4$ sessions, $>300$ minutes total) per quit attempt, leading to high quit rates demonstrating high clinical significance in a variety of typical clinical practice settings.

Emerging evidence is also now showing that 1:1 telemedicine (ie, audiovisual, Health Insurance Portability and Accountability Act compliant) tobacco cessation counseling is equally effective to quitline counseling, with higher participant satisfaction and medication compliance. ${ }^{52}$ Additionally, a recent telehealth videoconferencing group tobacco program has also shown feasibility with comparable quit rates to face-to-face group treatment in rural and urban areas of Canada. ${ }^{53}$ Thus, future research studies could examine group tobacco treatment mechanisms delivered via a telemedicine format more systematically, particularly in rural areas where face-to-face group treatment is difficult to establish. Finally, there is an urgent need for additional research on culturally sensitive group interventions. Webb Hooper et al. ${ }^{54}$ have shown initial promising results with a culturally tailored intervention on early quit rates for lower income Black tobacco users.

\section{US Health Care Systems Change and Policy Recommendations}

Several additional rationales beyond quit rates support offering group treatment. First, patient and clinician satisfaction remains high in group tobacco treatment in many cross-cultural settings. ${ }^{28,39}$ Therefore, patient experience or preference for treatment needs to remain a key factor in the referral process. We acknowledge that some individuals will always prefer individual, face-to-face, telephonic treatments or other nongroup options. Electronic or fax-toquit referral could be implemented more widely (eg, from primary care physicians and other medical specialists) to outpatient tobacco treatment clinics incorporating group treatment formats. These referrals could also be initiated directly from inpatient tobacco treatment programs, which are rapidly increasing in US hospitals, ${ }^{55}$ partly due to new Joint Commission standards. ${ }^{56}$

Second, group treatment is a standard of care for other substance use disorders (alcohol and illicit drugs).22,57 Many of these patients also use tobacco. Prior successful encounters in group treatment may increase these patients' receptivity to a group tobacco treatment modality due to prior earlier experiences in developing mastery skills and social identity change experiences acquired in similar recovery intervention formats..$^{58,59}$

Third, evidence-based worksite wellness tobacco treatment group programs have a strong potential to increase engagement in tobacco use treatment for those who are not reached in clinical settings. ${ }^{60}$ As indicated in Supplementary Appendix A, a sample closed 6-week group treatment protocol (adapted from an outpatient tobacco treatment clinic), demonstrated high attendance and high quit rates. ${ }^{23,27,39,60}$ This type of program would be somewhat comparable with worksite weight management wellness programs. Supplementary Appendix B includes examples of billable, clinic-based group reimbursable current procedural terminology codes, which can make tobacco cessation services sustainable in health care settings. Group treatment can be administered by clinicians such as Certified Tobacco Treatment Specialists, who are likely to implement high-fidelity group treatment protocols. These clinicians are trained in the core competencies outlined by the Association for the Treatment of Tobacco Use and Dependence (www.attud.org) through programs accredited by the Council for Tobacco Treatment Training Programs (www.ctttp.org).

Finally, there is growing evidence that while group treatment is likely to be at least as effective as individual treatment, it is more cost-effective than individual treatment. ${ }^{61-63}$ Ensuring access to group treatment may therefore result in reducing the cost burden on health care systems, insurers, and other payers (eg, businesses who self-insure). Also, because tobacco treatment is ranked one of the most cost-effective treatments of all preventive interventions, ${ }^{64}$ widespread tobacco group treatment implementation in health care settings would clearly reduce health care costs nationally, consistent with the Triple Aim of population health, ${ }^{65}$ now incorporated within the Affordable Care Act. ${ }^{66}$ Because of the ratio of clinicianto-patient time, group treatment is more efficient than individual treatment and, therefore, likely significantly more cost-effective. Due to the relationship between number of visits and outcomes, co-pays should not be added for each treatment visit. Creative reimbursement methods could be used with Medicare policies to encourage group treatment participation. Providing sufficient coverage and reimbursement for group treatment would place Medicare on par with newer, updated ${ }^{15}$ evidence-based treatment formats now mandated within private insurance and Medicaid plans. This will remain vital to realizing the high cost-effective benefits with many Medicare tobacco users accessing the new lung cancer screening programs seeking effective tobacco treatment services. .7,68 $^{6}$

\section{Conclusion}

Based on the available data from RCTs, meta-analyses, and typical clinical practice experiences in this narrative review, group treatment for tobacco dependence is at least as effective as-and possibly more effective than-individual counseling. The added advantages of efficiency and thus cost-effectiveness make this modality preferable. These results also demonstrate that group treatment is clearly feasible in various settings with good results. Thus, group treatment could and should be established and available in all behavioral health and medical settings.

\section{Supplementary Material}

Supplementary data are available at Nicotine \& Tobacco Research online.

\section{Funding}

The manuscript was not supported by any specific grant from any funding agency, commercial, or not-for-profit sectors.

\section{Declaration of Interests}

Chris Kotsen has no conflicts of interest to report with respect to this manuscript. He was a consultant to Infusion Pharma Consulting LLC in 2017. At the time of this research, Melissa Santorelli's salary was funded by the New Jersey 
Department of Health and the Centers for Disease Control and Prevention at the US Department of Health and Human Services under Cooperative Agreement Numbers NU58DP004822-03-1 and NU58DP003931-04-00. The contents of this publication are solely the responsibility of the authors and do not necessarily represent the official views of the New Jersey Department of Health, the United States Department of Health and Human Services, or the Centers for Disease Control and Prevention. She is currently employed by Merck \& Co., Inc. Erika Bloom is supported by a grant from the National Institutes on Drug Abuse (K23DA035288). She has been a consultant for WayBetter, Inc. Adam Goldstein has no conflicts of interest to report. Carol Ripley-Moffitt has no conflicts of interest to report. Michael Steinberg is a consultant for Arena Pharmaceuticals and an on-going consultant to Major League Baseball regarding tobacco policies. Michael Burke has worked on an unrestricted educational grant from Pfizer and an advisory board with GSK. He has no conflicts to report with respect to the topic of this manuscript. Jonathan Foulds is primarily funded by grants for the US National Institutes of Health (NIDA). He has done paid consulting work for pharmaceutical companies involved in manufacturing of smoking cessation products (eg, Pfizer, GSK) and has received a grant and study product from Pfizer.

\section{Acknowledgments}

We would like to personally thank Jennifer Greyber for editorial assistance with this manuscript. We would like to thank Chris Duffy and Kyle Downey for assisting with the PubMed search. We would also like to thank Maia Szulik, who provided assistance with the initial literature search. We would also like to acknowledge the support of the Association for the Treatment of Tobacco Use and Dependence (ATTUD) Business Practices Committee.

\section{References}

1. Office of the Surgeon General. The Health Consequences of Smoking-50 Years of Progress: A Report of the Surgeon General, 2014 I Surgeon General. gov. Rockville, MD: US Department of Health and Human Services; 2014.

2. Xu X, Bishop EE, Kennedy SM, Simpson SA, Pechacek TF. Annual healthcare spending attributable to cigarette smoking: an update. Am J Prev Med. 2015;48(3):326-333.

3. Babb S. Quitting Smoking Among Adults-United States, 2000-2015. MMWR Morb Mortal Wkly Rep. 2017;65(52):1457-1464.

4. Jamal A, Shanta D, Ann M, Lauren S, Martha E. Tobacco Use Screening and Counseling During Physician Office Visits Among Adults-National Ambulatory Medical Care Survey and National Health Interview Survey, United States, 2005-2009 (Supplement). Atlanta, GA: Centers for Disease Control and Prevention; 2012.

5. Chaiton M, Diemert L, Cohen JE, et al. Estimating the number of quit attempts it takes to quit smoking successfully in a longitudinal cohort of smokers. BMJ Open. 2016;6(6):e011045.

6. Borland R, Partos TR, Yong HH, Cummings KM, Hyland A. How much unsuccessful quitting activity is going on among adult smokers? Data from the International Tobacco Control Four Country cohort survey. Addiction. 2012;107(3):673-682.

7. Fiore M, Jaen C, Baker T, et al. Clinical Practice Guideline: Treating Tobacco Use and Dependence: 2008 Update. Rockville, MD: U.S. Department of Health and Human Services, Public Health Service. 2008.

8. Liu X, Li R, Lanza ST, Vasilenko SA, Piper M. Understanding the role of cessation fatigue in the smoking cessation process. Drug Alcohol Depend. 2013;133(2):548-555.

9. Yalom ID, Leszcz M. Theory and Practice of Group Psychotherapy. 5th ed. New York, NY: Basic Books; 2005.

10. Hajek P, Belcher M, Stapleton J. Enhancing the impact of groups: an evaluation of two group formats for smokers. Br J Clin Psychol. 1985;24(pt 4):289-294.

11. Hajek $\mathrm{P}$. Withdrawal-oriented therapy for smokers. $\mathrm{Br} J$ Addict. 1989;84(6):591-598.
12. Kanzler M, Jaffe JH, Zeidenberg P. Long- and short-term effectiveness of a large-scale proprietary smoking cessation program. -A 4-year follow-up of Smokenders participants. J Clin Psychol. 1976;32(3):661-669.

13. Bakkevig O, Steine S, von Hafenbrädl K, Laerum E. Smoking cessation. A comparative, randomised study between management in general practice and the behavioural programme SmokEnders. Scand J Prim Health Care. 2000;18(4):247-251.

14. Lando HA, McGovern PG, Barrios FX, Etringer BD. Comparative evaluation of American Cancer Society and American Lung Association smoking cessation clinics. Am J Public Health. 1990;80(5):554-559.

15. Siu AL; U.S. Preventive Services Task Force. Behavioral and pharmacotherapy interventions for tobacco smoking cessation in adults, including pregnant women: U.S. preventive services task force recommendation statement. Ann Intern Med. 2015;163(8):622-634.

16. Mottillo S, Filion KB, Bélisle P, et al. Behavioural interventions for smoking cessation: a meta-analysis of randomized controlled trials. Eur Heart J. 2009;30(6):718-730.

17. Stead LF, Carroll AJ, Lancaster T. Group behaviour therapy programmes for smoking cessation. Cochrane Database Syst Rev. 2017;3:CD001007.

18. Departments of Health and Human Services, Labor, and the Treasury. Frequently Asked Questions About Affordable Care Act Implementation (Part XIX). Baltimore, MD: Centers for Medicare \& Medicaid Services; 2014.

19. Baumeister RF, Leary MR. Writing narrative literature reviews. Rev Gen Psychol. 1997;1(3):311-320.

20. Green BN, Johnson CD, Adams A. Writing narrative literature reviews for peer-reviewed journals: secrets of the trade. J Chiropr Med. 2006;5(3):101-117.

21. Foulds J, Steinberg MB, Williams JM, Ziedonis DM. Developments in pharmacotherapy for tobacco dependence: past, present and future. Drug Alcohol Rev. 2006;25(1):59-71.

22. Williams JM, Steinberg ML, Kenefake AN, Burke MV. An argument for change in tobacco treatment options guided by the ASAM criteria for patient placement. J Addict Med. 2016;10(5):291-299.

23. Steinberg MB, Foulds J, Richardson DL, Burke MV, Shah P. Pharmacotherapy and smoking cessation at a tobacco dependence clinic. Prev Med. 2006;42(2):114-119.

24. Roberts E, Eden Evins A, McNeill A, Robson D. Efficacy and tolerability of pharmacotherapy for smoking cessation in adults with serious mental illness: a systematic review and network meta-analysis. Addiction. 2016;111(4):599-612.

25. West R. Assessing smoking cessation performance in NHS Stop Smoking Services: The Russell Standard (Clinical); 2005. http://www.ncsct.co.uk/ usr/pub/assessing-smoking-cessation-performance-in-nhs-stop-smokingservices-the-russell-standard-clinical.pdf.

26. Hiscock R, Murray S, Brose LS, et al. Behavioural therapy for smoking cessation: The effectiveness of different intervention types for disadvantaged and affluent smokers. Addict Behav. 2013;38(11):2787-2796.

27. Foulds J, Gandhi KK, Steinberg MB, et al. Factors associated with quitting smoking at a tobacco dependence treatment clinic. Am J Health Behav. 2006;30(4):400-412.

28. Dobbie F, Hiscock R, Leonardi-Bee J, et al. Evaluating long-term outcomes of NHS Stop Smoking Services (ELONS): a prospective cohort study. Health Technol Assess. 2015;19(95):1-156.

29. Judge K, Bauld L, Chesterman J, Ferguson J. The English smoking treatment services: short-term outcomes. Addiction. 2005;100(suppl 2):46-58.

30. McEwen A, West R, McRobbie H. Effectiveness of specialist group treatment for smoking cessation vs. one-to-one treatment in primary care. Addict Behav. 2006;31(9):1650-1660.

31. Bauld L, Ferguson J, Lawson L, Chesterman J, Judge K. Tackling Smoking in Glasgow: Final Report. 2005/11, Revised January 2006. Glasgow, Scotland: University of Glasgow; 2006.

32. Bauld L, Chesterman J, Ferguson J, Judge K. A comparison of the effectiveness of group-based and pharmacy-led smoking cessation treatment in Glasgow. Addiction. 2009;104(2):308-316. 
33. Brose LS, West R, McDermott MS, Fidler JA, Croghan E, McEwen A. What makes for an effective stop-smoking service? Thorax. 2011;66(10):924-926.

34. Mardle T, Merrett S, Wright J, Percival F, Lockhart I. Real world evaluation of three models of NHS smoking cessation service in England. BMC Res Notes. 2012;5:9.

35. McAlpine K, Kośmider L, Anastasi N, Rossabi J. Effectiveness of smoking cessation services in England-Clinic in Whitechapel as an example. Przegl Lek. 2015;72(10):496-499.

36. Nolan MB, Warner DO. Perioperative tobacco use treatments: putting them into practice. BMJ. 2017;358:j3340.

37. Bloom EL, Burke MV, Kotsen C, et al. Billing practices among U.S. tobacco use treatment providers. J Addict Med. In press.

38. American Conference on Diversity. New Jersey: A Statewide View of Diversity. A Report on the Leadership Conference on Diversity Issues Impacting New Jersey. American Conference on Diversity IfRaCT, Leadership New Jersey, and New Jersey Public Policy Research Institute. New Brunswick, NJ: Rutgers, The State University of New Jersey, Edward J. Bloustein Center for Survey Research; 2007.

39. UMDNJ-School of Public Health. Tobacco Surveillance Data Brief: A Profile of New Jersey Quitcenters. Piscataway NJ: University of Medicine \& Dentistry-School of Public Health; 2007.

40. Sutherland G, Stapleton JA, Russell MA, et al. Randomised controlled trial of nasal nicotine spray in smoking cessation. Lancet. 1992;340(8815):324-329.

41. Anthonisen NR, Connett JE, Kiley JP, et al. Effects of smoking intervention and the use of an inhaled anticholinergic bronchodilator on the rate of decline of FEV1. The Lung Health Study. JAMA. 1994;272(19): 1497-1505.

42. Anthonisen NR, Skeans MA, Wise RA, Manfreda J, Kanner RE, Connett JE; Lung Health Study Research Group. The effects of a smoking cessation intervention on 14.5-year mortality: a randomized clinical trial. Ann Intern Med. 2005;142(4):233-239.

43. Connett JE, Kusek JW, Bailey WC, O'Hara P, Wu M. Design of the Lung Health Study: a randomized clinical trial of early intervention for chronic obstructive pulmonary disease. Control Clin Trials. 1993;14(suppl 2):3S-19S

44. Hall SM, Humfleet GL, Muñoz RF, Reus VI, Robbins JA, Prochaska JJ. Extended treatment of older cigarette smokers. Addiction. 2009;104(6):1043-1052.

45. Hall SM, Humfleet GL, Muñoz RF, Reus VI, Prochaska JJ, Robbins JA. Using extended cognitive behavioral treatment and medication to treat dependent smokers. Am J Public Health. 2011;101(12):2349-2356.

46. Song F, Maskrey V, Blyth A, et al. Differences in longer-term smoking abstinence after treatment by specialist or Nonspecialist advisors: secondary analysis of data from a relapse prevention trial. Nicotine Tob Res. 2016;18(5):1061-1066.

47. de Bruin M, Viechtbauer W, Eisma MC, et al. Identifying effective behavioural components of Intervention and Comparison group support provided in SMOKing cEssation (IC-SMOKE) interventions: a systematic review protocol. Syst Rev. 2016;5:77.

48. Goldstein AO, Gans SP, Ripley-Moffitt C, Kotsen C, Bars M. Use of expired air carbon monoxide testing in clinical tobacco treatment settings. Chest. 2018;153(2):554-562.

49. West R, Evans A, Michie S. Behavior change techniques used in groupbased behavioral support by the English stop-smoking services and preliminary assessment of association with short-term quit outcomes. Nicotine Tob Res. 2011;13(12):1316-1320.
50. Stead LF, Lancaster T. Combined pharmacotherapy and behavioural interventions for smoking cessation. Cochrane Database Syst Rev. 2012;10:CD008286.

51. Public Health England. Models of delivery for stop smoking services: Options and evidence. London, England: PHE Publications; 2017].

52. Richter KP, Shireman TI, Ellerbeck EF, et al. Comparative and cost effectiveness of telemedicine versus telephone counseling for smoking cessation. J Med Internet Res. 2015;17(5):e113.

53. Carlson LE, Lounsberry JJ, Maciejewski O, Wright K, Collacutt V, Taenzer P. Telehealth-delivered group smoking cessation for rural and urban participants: Feasibility and cessation rates. Addict Behav. 2012;37(1):108-114.

54. Webb Hooper M, Antoni MH, Okuyemi K, Dietz NA, Resnicow K. Randomized controlled trial of group-based culturally specific cognitive behavioral therapy among African American smokers. Nicotine Tob Res. 2017;19(3):333-341.

55. Nahhas GJ, Wilson D, Talbot V, et al. Feasibility of implementing a hospital-based "Opt-Out" tobacco-cessation service. Nicotine Tob Res. 2017;19(8):937-943.

56. Fiore MC, Goplerud E, Schroeder SA. The Joint Commission's new tobacco-cessation measures-will hospitals do the right thing? N Engl J Med. 2012;366(13):1172-1174.

57. Mee-Lee D. The ASAM Criteria: Treatment for Addictive, Substancerelated, and Co-occurring Conditions. Rockville, MD: American Society of Addiction Medicine; 2013.

58. Prochaska JJ, Delucchi K, Hall SM. A meta-analysis of smoking cessation interventions with individuals in substance abuse treatment or recovery. $J$ Consult Clin Psychol. 2004;72(6):1144-1156.

59. Best D, Beckwith M, Haslam C, et al. Overcoming alcohol and other drug addiction as a process of social identity transition: the social identity model of recovery (SIMOR). Addict Res Theory. 2016;24(2):111-123.

60. Cahill K, Lancaster T. Workplace interventions for smoking cessation. Cochrane Database Syst Rev. 2014(2):CD003440.

61. Boyd KA, Briggs AH. Cost-effectiveness of pharmacy and group behavioural support smoking cessation services in Glasgow. Addiction. 2009;104(2):317-325.

62. Bauld L, Boyd KA, Briggs AH, et al. One-year outcomes and a cost-effectiveness analysis for smokers accessing group-based and pharmacy-led cessation services. Nicotine Tob Res. 2011;13(2):135-145.

63. Prochaska JJ, Benowitz NL. The past, present, and future of nicotine addiction therapy. Annu Rev Med. 2016;67:467-486.

64. Maciosek MV, LaFrance AB, Dehmer SP, et al. Health benefits and cost-effectiveness of brief clinician tobacco counseling for youth and adults. Ann Fam Med. 2017;15(1):37-47.

65. Berwick DM, Nolan TW, Whittington J. The triple aim: care, health, and cost. Health Aff (Millwood). 2008;27(3):759-769.

66. Whittington JW, Nolan K, Lewis N, Torres T. Pursuing the triple aim: the first 7 years. Milbank Q. 2015;93(2):263-300.

67. Park ER, Gareen IF, Japuntich S, et al. Primary care provider-delivered smoking cessation interventions and smoking cessation among participants in the National Lung Screening Trial. JAMA Intern Med. 2015;175(9):1509-1516.

68. Fucito LM, Czabafy S, Hendricks PS, Kotsen C, Richardson D, Toll BA; Association for the Treatment of Tobacco Use and Dependence/Society for Research on Nicotine and Tobacco Synergy Committee. Pairing smoking-cessation services with lung cancer screening: a clinical guideline from the Association for the Treatment of Tobacco Use and Dependence and the Society for Research on Nicotine and Tobacco. Cancer. 2016;122(8):1150-1159. 